\title{
Alcuni elementi di meccanica negli spazi curvi.
}

\author{
Memoria di Gruseppe Vitali (a Bologna).
}

Sunto. - L'A. espone detti elementi col metodo della rappresentazione funcionale, metodo che, come egli dice, nella sostanza equivale al metodo vettoriale, ma nella forma lo supera per la scorrevolezza e per la naturalezza. L'A. approfitta della pubblicazione per sottolineare alcune asservazioni, delle quali pensa di dover trar partito in altra occasione.

Non mi provo ad indicare l'ultimo fine scientifico a cui tendo con questa succinta pubblicazione. Penso che ora non avrei tutti gli elementi per esprimermi in modo chiaro. Posso soltanto dire che la definizione che figura nel $\S 3$ ed il contenuto del $\$ 5$ costituiscono del materiale che richiameró in altra occasione.

Per ora basta che l'attenzione del lettore si soffermi sul fine immediato e modesto di questa pubblicazione, che è l'esposizione semplice, malgrado il punto di vista tanto generale, di alcuni elementi della meccanica.

Come in altre questioni, anche qui la semplicità è ottenuta col metodo della rappresentazione funzionale di cui ho dato parecchi saggi nello studio della geometria $\left({ }^{1}\right)$.

Questo metodo è equivalente nella sostanza al metodo vettoriale, ma lo supera nella forma per la scorrevolezza e per la naturalezza.

La nota si compone di 5 paragrafi. Nel $\S 1$ si parla di vettori e di campo di vettori in una varietà ad $n$ dimensioni, e si esaminano gli elementi analitici che servono ad individuarli.

Nel $\$ 2$ si esamina il moto di un punto in una varietà ad $n$ dimensioni e se ne trae la nozione di accelerazione.

Nel $\$ 3$ si considerano i campi di forze e le loro azioni sui punti materiali. Qui si vuol seguire la concezione classica. Però si è indotti ad attribuire ad ogni punto materiale ed in relazione a ciascun campo di forze nel quale sia immerso, due masse, che io chiamo massa inerte e massa attiva.

(1) G. VITALI, Geometria nello spazio Hibertiano, (Zanichelli, Bologna 1929). Citerò nel seguito questo libro colla sigla «G.H. ". 
Nel $\S 4$ si dimostra un teorema che in circostanze particolari puó agevolare la risoluzione del problema del moto di un punto materiale in presenza di un campo di forze. Se ne fa l'applicazione allo studio del moto nello spazio euclideo a tre dimensioni, quando le forze del campo siano tutte dirette verso un medesimo punto 0 ed infine siano proporzionali inversamente al quadrato della distanza del punto di applicazione da 0.

Nel $\S 5$ si immagina che lo spazio ambiente sia una ipersfera di uno spazio lineari a 4 dimensioni, si affronta in questo spazio sferico il problema analogo a quello particolare trattato nel paragrafo precedente. Ne viene che, postulando l'azione reciproca di due corpi materiali in modo analogo al consueto (legge di NewToN), le leggi del moto sono come le consuete, non si verificherebbero spostamenti dei perieli dei pianeti. Una postulazione più fantasiosa di detta azione reciproca, potrebbe però portare a leggi che implicherebbero tali spostamenti, e con particolare determinazione di costanti si potrebbe ottenere quello che si ritiene essere lo spostamento del perielio di Mercurio.

\section{\& 1. Vettori.}

1. DeF. Se $V_{n}$ è una varietà ad $n$ dimensioni, se $P$ è un punto di $V_{n}$, se $F$ è un parametro $\left(^{1}\right)$ di una direzione taugente a $V_{n}$ in $P$, si dirà che $F$ è un vettore di $V_{n}$ uscente da $P$.

2. Sia

una determinante di $V_{n}\left({ }^{2}\right)$.

$$
f(t ; u) \text { od } f(u)
$$

Evidentemente se $P$ è un punto di $V_{n}$, e se $F$ è un vettore di $V_{n}$ uscente da $P$, si avra

$$
F=\Sigma_{i} f_{i} F^{i}
$$

dove le $F^{i}$ sono delle convenienti costanti rispetto a $t$, e che per le sostituzioni invertibili sulle variabili $u$ si comportano come gli elementi di un controvariante ad un apice di classe $1\left(^{3}\right)$.

Si ponga

$$
F_{j}=\sum_{1}^{n} a_{i, j} F^{i}\left({ }^{4}\right)
$$

(1) " G. H. ", p. 87.

$\left({ }^{2}\right)$ * G. H. », p. 85.

(3) « G. H. ", p. $15 \tilde{\text { o. }}$

$\left({ }^{4}\right)$ * G. H. », p. 181. 
Si ha subito

ed anche

$$
F_{j}=\int_{g}\left(\Sigma_{i} f_{i} F^{i}\right) f_{j} d t=\int_{g} F f_{j} d t
$$

$$
F^{i}=\Sigma_{j} a_{1}^{i, j} F_{j}\left({ }^{1}\right)
$$

Infine si può aggiungere che le $F_{j}$ si comportano, per le sostituzioni invertibili sulle variabili $u$, come un covariante ad un indice di classe 1.

DeF. Le $F^{i}$ si dicono le componenti controvarianti del vettore $F$, e le $F_{3}$ si dicono le componenti covarianti del vettore $F$.

Le formule precedenti provano che gli elementi:

\section{$1 .^{\circ}$ Vettore}

2.0 Componenti controvarianti del vettore

3. Componenti covarianti del vettore

sollo tutti noti quando se ne conosca uno.

3. Def. Quando per ogni punto della varietà $V_{n}$ è dato un vettore si dice che si ha un campo di vettori.

Le componenti dei vettori di un campo risultano allora funzioni delle $n$ variabili $u$.

Talvolta ci capiterà di considerare un campo di vettori variabile col tempo. Se $\tau$ indica la misura del tempo, allora le componenti dei vettori risulteranno oltre che funzioni delle $u$ anche funzioni $\mathrm{di} \tau$.

4. Def. Un campo di vettori indipendente dal tempo si dice conservativo. se, essendo $F_{j}$ le sue componenti covarianti, la

$$
\Sigma_{j} F_{j} d u_{j}
$$

è un differenziale esatto; e la funzione $U$ di cui le $F_{j}$ sono le derivate parziali si dice il potenziale del campo di forze.

\section{§ 2. Accelerazione.}

1. Il moto di un punto in $V_{n}$ si ha dando le $u_{i}$ in fumzione di $\tau$. Supponiamo che sia

$$
u_{i}=u_{i}(\tau) \quad(i=1,2, \ldots, n)
$$

(1) * G. H. », p. 181. 
Allora la $f(u)$ diventa una funzioue di $\tau$ che indicheremo con $\varphi(\tau)$, la quale descriverà una curva $C$.

Indicando con accenti le derivate prime e seconde rispetto a $\tau$ nel punto $P$, si ha

$$
\begin{aligned}
\varphi^{\prime} & =\Sigma_{i} f_{i} u_{i}^{\prime} \\
\varphi^{\prime \prime} & \left.=\Sigma_{r s} f_{r,} u_{s} u_{1}^{\prime} u_{s}^{\prime}+\Sigma_{i} f_{i}\left(u_{i}^{\prime \prime}+\Sigma_{r s} G_{r s}^{i} u_{q}^{\prime} u_{s}^{\prime}\right){ }^{1}\right)
\end{aligned}
$$

dove il simbolo di Christoffer è di classe 1.

2. Consideriamo un valore di $\tau$ ed indichiamo con $P$ il punto di $C$ in cui si trova il mobile nell' istante $\tau$ e con $\gamma$ la geodetica $\left(^{2}\right)$ di $V_{n}$ tangente a $C$ in $P$.

Supponiamo che a partire da $P$ il mobile prosegua il suo movimento su $\gamma$ con moto uniforme e di velocita uguale a quella che il mobile ha raggiunto in $P$. Per questo moto le $u_{i}$ risulteramo certe funzioni di $\tau$, che saranno in generale diverse dalle (1), e che noi indicheremo con

$$
u_{i}=w_{i}(\tau) \quad(i=1,2, \ldots, n) .
$$

Corrispondentemente la $f(u)$ diventerà una punto-funzione di $\tau$ che indicheremo con $\psi(\tau)$.

Indicando sempre con accenti le derivate prime e seconde rispetto a $\tau$, nel punto $P$, si avrà

$$
w_{i}^{\prime}=u_{i}^{\prime},
$$

ma in generale non saranno uguali le $w_{i}^{\prime \prime}$ ed $u_{i}^{\prime \prime}$.

Se $\sigma$ indica la lunghezza di arco della $\gamma$, essendo $\gamma$ una geodetica di $V_{n}$, si avrà luıgo $\gamma$

$$
\frac{d^{2} w_{i}}{d \sigma^{2}}+\Sigma_{r s} C_{r s}^{i} \frac{d w_{r}}{d \sigma} \cdot \frac{d w_{s}}{d \sigma}=0 \quad(i=1,2, \ldots, n)\left({ }^{3}\right) .
$$

In particolare la $(5)$ varrà nel punto $P$.

Se inoltre $v$ è la grandezza della velocità lungo $\gamma$, ossia se

si avrà lıngo $\gamma$,

e quindi, per la (5), si avrà

$$
v^{2}=\Sigma_{r s} a_{r, s} u_{s}^{\prime} u_{s}^{\prime}
$$

$$
d \sigma=v \cdot d \tau
$$

$$
w_{i}^{\prime \prime}+\Sigma_{r_{s}} C_{r s}^{i} w_{r}^{\prime} w_{s}^{\prime}=0 \quad(i=1,2, \ldots, n)
$$

(1) «G. H. *, pp. 201, 202 .

(2) \& G. H. », p. 226.

(3) «G. H. », p. 228. 
poichè per la (7) il primo membro della (8) vale il primo membro della (5) moltiplicato per $v^{2}$.

Tenendo conto delle (4) e delle (8), si ha nel punto $P$

e dalle (3) e $\left(3^{\prime}\right)$ si ha

$$
\begin{gathered}
\psi^{\prime}=\varphi^{\prime}, \\
\psi^{\prime \prime}=\Sigma_{r_{s}} f_{r, s}^{\prime} u v_{r}^{\prime} u_{s}^{\prime},
\end{gathered}
$$

$$
\varphi^{\prime \prime}=\psi^{\prime \prime}+\Sigma_{i} f_{i}\left(u_{i}^{\prime \prime}+\Sigma_{r s} C_{r s}^{i} u_{r}^{\prime} u_{s}^{\prime}\right) .
$$

Osserviamo inoltre che nel punto $P$ è

$$
\varphi=\psi \text {. }
$$

3. Si ha allora, trascurando gli infinitesimi dí ordine superiore al secondo,

$$
\begin{aligned}
& \varphi(\tau+d \tau)=\varphi(\tau)+\varphi^{\prime}(\tau) d \tau+\frac{1}{2} \varphi^{\prime \prime}(\tau) d \tau^{2} \\
& \psi(\tau+d \tau)=\psi(\tau)+\psi^{\prime}(\tau) d \tau+\frac{1}{2} \psi^{\prime \prime}(\tau) d \tau^{2}
\end{aligned}
$$

e, tenendo conto delle $\left(2^{\prime}\right),\left(3^{\prime \prime}\right)$ e $(9)$, si ha

$$
\varphi(\tau+d \tau)-\psi(\tau+d \tau)=\frac{1}{2} \Sigma_{i} f_{i}\left(u_{i}^{\prime \prime}+\Sigma_{1 \cdot s} C_{r s}^{i} u_{r}^{\prime} u_{s}^{\prime}\right) d \tau^{2}
$$

4. Il parametro che figura nel secondo membro di (10) individua una direzione orientata dello spazio tangente in $P$ a $V_{n}$, la quale direzione è indipendente dal valore di $d \tau$.

Uno dei parametri di tale direzione orientata è

$$
\Sigma_{i} f_{i}\left(u_{i}^{\prime \prime}+\Sigma_{r s} C_{r s}^{i} u_{s}^{\prime} u_{s}^{\prime}\right) \text {. }
$$

DEF. Il parametro (11) è un vettore della $V_{n}$ che diremo l'accelerazione del punto mobile nell' istante $\tau$ (relativa all' unità di tempo scelta).

Se con $A^{j}$ si indicano le componenti controvarianti di questa accelerazione e con $A$, le sue componenti covarianti, si ha subito

$\mathrm{e}$

$$
A^{j}=u_{j}^{\prime \prime}+\Sigma_{r s} C_{r s}^{j} u_{r}^{\prime} u_{s}^{\prime}
$$

$$
A_{r}=\mathrm{\Sigma}_{j} a_{r, j} A^{j} \text {. }
$$

\section{$\S$ 3. Campo di forze.}

1. DeF. Un campo di vettori $F$, di componenti controvarianti $F^{j}$, si dice un campo di forze, quando rappresenta uno stato fisico della varietà $V_{n}$, 
corrispondentemente al quale ad ogni punto materiale vengono associati due numeri $m_{i}$ ed $m_{a}\left({ }^{1}\right)$ che chiamerò massa inerte e massa attiva, tali che per effetto del campo di forze il punto materiale debba muoversi in modo che siano soddisfatte le relazioni

$$
m_{i} A^{j}=m_{a} F^{j} \quad(j=1,2, \ldots, n)\left({ }^{2}\right),
$$

dove le $A^{j}$ sono le componenti controvarianti dell'accelerazione. Le (1) si possono anche scrivere

$$
m_{i} A_{j}=m_{a} F_{j} \quad(j=1,2, \ldots, n) .
$$

2. Le (1) sono $n$ equazioni differenziali ordinarie del secondo ordine sopra $n$ funzioni incognite $u_{j}$ della variabile $\tau$, quindi esse individuano una soluzione quando di questa soluzione sono dati i valori iniziali delle $u_{j}$ e delle loro derivate.

In altri termini un campo di forza determina in modo unico il moto di un punlo materiale quando di questo punto si conosca la posizione $e$ la velocitd iniziale.

3. Derivando i due membri della

$$
v^{2}=\Sigma_{r s} a_{r, s} u_{r}^{\prime} u_{s}^{\prime}
$$

applicando la regola di derivazione assoluta lungo una curva dei sistemi composti $\left({ }^{3}\right)$, e dividendo per 2 , si ottiene

$$
v \cdot v^{\prime}=\Sigma_{j,}, a_{j, r} \cdot u_{r}{ }^{\prime}\left(u_{j}^{\prime \prime}+\Sigma_{h k} C_{h k}^{j} u_{h}{ }^{\prime} u_{k}{ }^{\prime}\right)=\Sigma_{j r} a_{j,}, u_{r}{ }^{\prime} A^{j}=\Sigma_{r} u_{r}{ }^{\prime} A_{r},
$$

e moltiplicando per $m_{i}$, e tenendo conto delle $\left(1^{\prime}\right)$ si ha

ed infine

$$
m_{i} \cdot v \cdot v^{\prime}=m_{a} \cdot \Sigma_{r} F_{r} u_{r}^{\prime}
$$

$$
d \frac{m_{i} \cdot v^{2}}{2}=m_{a} \cdot \Sigma_{r} F_{r} d u_{r}
$$

4. DrF. L'espressione

$$
\frac{m_{i} \cdot v^{2}}{2}
$$

(1) Determinati all'infuori di un medesimo fattore.

(2) Se, per esempio, il eampo di forze è quello generato dalla presenza di una massa materiale, per il nostro punto materiale si ha: $m_{i}=m_{\alpha}=$ massa materiale del punto. Se il campo è generato da una massa elettrica, per il punto materiale $m_{i}$ è la massa materiale ed $m_{a}$ ̀े la sua massa elettrica.

(3) « G. H. », p. 225. 
si dice for $z a$ viva, e l'espressione

si chiama lavoro elementare.

$$
m_{a} \cdot \Sigma_{r} F_{r} d u_{r}
$$

Per la (2) si ha allora il

Teor. Il differenziale della forza viva è uguale al lavoro elementare.

5. Se il campo di forze è conservativo, e se $U$ è il suo potenziale, le equazioni (1') diventano

e, più per disteso,

$$
m_{i} A_{j}=m_{a} \cdot \frac{\partial U}{\partial u_{j}} \quad(j=1,2, \ldots, n)
$$

$$
m_{i}\left[\Sigma_{r} a_{r, j} u_{r}^{\prime \prime}+\Sigma_{r k} a_{s \cdot k, j} u u_{r}^{\prime} u_{k}^{\prime}\right]=m_{a} \frac{\partial U}{\partial u_{j}} \quad(j=1,2, \ldots, n) .
$$

La (2) diventa

$$
d \frac{m_{i} \cdot v^{2}}{2}=m_{a} d U
$$

che, integrando, dà

$$
\frac{m_{i} \cdot v^{2}}{2}=m_{a} U+\text { costante. }
$$

\section{Poniamo}

$$
L=\frac{m_{i} \cdot v^{2}}{2}+m_{a} \cdot v
$$

Le (3). si possono compendiare nella formula variazionale

$$
\delta \int L d \tau=0
$$

per tutte le variazioni delle $u$ nulle agli estremi di integrazione.

Infatti le equazioni di EuLERo sono

e, poichè

$$
\frac{d}{d \tau} \frac{\partial L}{\partial u_{j}^{\prime}}-\frac{\partial L}{\partial u_{j}}=0
$$

$$
\frac{d}{d \tau} \frac{\partial L}{\partial u_{j}^{\prime}}=m_{i} \cdot \frac{d}{d \tau} \Sigma_{r} \cdot a_{r, j} u_{r}^{\prime}=m_{i}\left[\Sigma_{r}, a_{r, j} u_{r}^{\prime \prime}+\Sigma_{r_{k}}\left(a_{\vartheta \cdot k, j}+a_{r, j k}\right) u_{r}^{\prime} u_{k}^{\prime}\right]
$$

e

$$
\frac{\partial L}{\partial u_{j}}=m_{i} \Sigma_{r k} a_{r, i k} u_{r}^{\prime} u_{k}^{\prime}+m_{a} \frac{\partial U}{\partial u_{j}}
$$

si vede che le (7) diventano appunto le (3).

La formula variazionale (6) costituisce il principio di Hamuton nella $V_{n}$. 


\section{§ 4. Sul moto per particolari campi di forze.}

1. DEF. Se $V$ è una varietà ad $n$ dimensioni, un'altra varietá $V^{\prime}$ ad $m$ dimensioni $(m<n)$ contenuta in $V$ si dice una vera varietà geodetica di $V$ se tutte le geodetiche di $V^{\prime}$ sono anche geodetiche di $V$.

2. Teor. Se $V$ è una varietà ad $n$ dimensioni che contiene una vera varietà geodetica $V^{\prime}$ ad $m$ dimensioni $(m<n)$, se $C$ è un campo di forze in $V$ tale che le sue forze applicate ai punti di $V^{\prime}$ siano tangenti a $V^{\prime}$, il moto di un punto sotto l'azione di $C$ si svolge tutto sopra $V^{\prime}$, se inizialmente il punto giace su $V^{\prime}$ e la sua velocità iniziale è tangente a $V^{\prime}$.

Dim. Supponiamo che la $V^{\prime}$ sia la

$$
u_{m+1}=u_{m+2}=\ldots=u_{n}=0 .
$$

Se $\sum_{1}^{n}{ }_{r s} a_{r, s} d u_{r} d u_{s}$ è il quadrato dell' elemento lineare, e se si indica con $a_{r, s}^{0}$ ció che diventa $a_{r, s}$ quando vi si faccia $u_{m+1}=0, u_{m+2}=0, \ldots, u_{n}=0$, il quadrato dell' elemento lineare di $V^{\prime}$ è dato da

$$
\sum_{1}^{m}{ }_{r s} a_{r, s}^{0} d u_{r} d u_{s}
$$

ed i relativi simboli di Christoffel, che indicheró con $K_{r s}^{j}$ si ottengono dai corrispondenti di $V$ facendo in essi $u_{m+1}=0, u_{m+2}=0, \ldots, u_{n}=0$.

Poichè tutte le geodetiche di $V^{\prime}$ sono geodetiche in $V$, e per queste geodetiche deve essere $u_{m+1}=0, u_{m+2}=0, \ldots, u_{n}=0$, e quindi $u_{p}^{\prime}=u_{p}^{\prime \prime}=0$ $(p=m+1, m+2, \ldots, n)$ le

diventano per esse

$$
u_{p}^{\prime \prime}+\sum_{1}^{n}{ }_{r s}^{p} C_{r, s}^{p} u_{r}^{\prime} u_{s}^{\prime}=0 \quad(p=m+1, m+2, \ldots, n),
$$

$$
\sum_{1}^{m} K_{r, s}^{p} u_{r}^{\prime} u_{s}^{\prime}=0 \quad(p=m+1, m+2, \ldots, n),
$$

e, poichè in un punto qualunque di $V^{\prime}$ una geodetica di $V^{\prime}$ può avere qualunque direzione, sarà

$$
K_{r, s}^{p}=0 \quad(r, s=1,2, \ldots, m)(p=m+1, m+2, \ldots, n) .
$$

Le equazioni del moto in $V$ sono

$$
m_{i}\left(u_{j}^{\prime \prime}+\sum_{1}^{n}{ }_{n s} C_{r s}^{j} u_{r}^{\prime} u_{s}^{\prime}\right)=m_{a} F^{j} .
$$


Evidentemente indicando con $G^{j}$ ciò che diventa $F^{j}$ quando vi si ponga $u_{m+1}=u_{m+2}=\ldots=u_{n}=0$, si ha

$$
G^{p}=0 \quad(p=m+1, m+2, \ldots, n) .
$$

Sostituiamo nelle (2) lo zero al posto delle

esse diventano

$$
u_{p}, u_{p}^{\prime}, u_{p}^{\prime \prime} \quad(p=m+1, m+2, \ldots, n)
$$

$$
m_{i}\left(u_{j}^{\prime \prime}+\sum_{1}^{m} K_{r s}^{j} K_{r s}^{j} u_{r}^{\prime} u_{s}^{\prime}\right)=m_{a} G^{j} \quad(j=1,2, \ldots, m),
$$

le ultime $n-m$ equazioni (2) risultando soddisfatte in virtù delle (1) e (3).

Ora le (4) hanno una soluzione determinata quando sono fissati i valori iniziali delle

Questa soluzione insieme con

$$
u_{j} \cdot u_{j}^{\prime} \quad(j=1,2, \ldots, m) \text {. }
$$

$$
u_{p}=0 \quad(p=m+1, m+2, \ldots, n)
$$

dà una soluzione di (2) che inizialmente passa per un punto di $V^{\prime}$ ed ha velocità iniziale tangente a $V^{\prime}$. E siccome questi elementi iniziali individuano la soluzione di (2) coi dati iniziali appartenenti a $V^{\prime}$, possiamo dire che una soluzione di (2) coi dati iniziali appartenenti a $V^{\prime}$ fornisce una linea giacente in $V^{\prime}$.

Oss. Consegue che tutte le volte che si ha un campo di forze in una varietà $V$, e i dati iniziali del moto sono contenuti in una vera varietá geodetica $V^{\prime}$ di $V$, la quale contenga inoltre tutte le forze del campo uscenti dai suoi punti, il problema del moto in $V$ si riduce ad un problema ana$\log _{0}$ in $V^{\prime}$.

3. Supponiamo che $n=3$, e che $V$ sia lo spazio euclideo a 3 dimensioni. Supponiamo poi che le forze di un campo $C$ (in $V$ ) siano dirette tutte verso un punto $O$ (di $V$ ).

Sia nota la posizione e la velocitá iniziale di un punto materiale. Questi elementi iniziali ed il punto $O$ individuano un piano $\Pi$, che, essendo una vera varieta geodetica della $V$, e contenendo tutte le forze di $C$ applicate ai suoi punti, deve contenere, pel teor. prec., la curva descritta dal mobile.

E la legge del movimento si può studiare su $\Pi$.

Per questo assumiamo su $\Pi$ un sistema di coordinate polari con polo in $O$. In tal caso il quadrato dell' elemento lineare di II è dato da

$$
d \rho^{2}+\rho^{2} d \theta^{2}
$$


Identificando $\rho$ con $u_{1}$ e $\theta$ con $u_{2}$, si trova

$$
a_{11,1}=a_{12,1}=a_{11,2}=a_{22,2}=0, \quad a_{12,2}=-a_{22,1}=p
$$

e conseguentemente

$$
C_{11}^{1}=C_{12}^{i}=C_{11}^{2}=C_{22}^{2}=0, \quad C_{12}^{2}=\frac{1}{\rho}, \quad C_{22}^{2}=-\rho .
$$

Inoltre, per l'ipotesi fatta sul campo di forza, è $F_{2}=0$.

Se poi noi supponiamo che la forza sia inversamente proporzionale al quadrato della distanza del punto di applicazione da 0 , avremo

$$
F^{1}=\frac{-k^{2}}{\rho^{2}} \quad(k=\text { costante reale })
$$

e poichè risulta inoltre

$$
F_{1}=\frac{-k^{2}}{\rho^{2}}, \quad F_{2}=0
$$

esiste un potenziale

$$
U=k^{2} \cdot \frac{1}{\rho}
$$

e si ha la relazione

$$
m_{i} \frac{v^{2}}{2}-m_{a} k^{2} \frac{1}{\rho}=K \quad(K=\text { costante reale })\left({ }^{1}\right)
$$

Inoltre una delle equazioni del moto è

$$
\theta^{\prime \prime}+2 \frac{\rho^{\prime} \theta^{\prime}}{\rho}=0\left({ }^{2}\right)
$$

da cui si ricava

0 , se si vuole,

$$
\log \theta^{\prime}+\log \rho^{2}=\text { costante }
$$

$$
\theta^{\prime} \rho^{2}=H
$$

$(H=$ costante $)$.

Ora, posto

$$
\zeta=\frac{1}{\rho}
$$

e quindi

si ha

$$
p^{\prime}=-p^{2} \zeta^{\prime}
$$

$$
v^{2}=\rho^{\prime 2}+\rho^{2} \theta^{\prime 2}=\rho^{+} \zeta^{\prime 2}+\rho^{2} \theta^{\prime 2},
$$

(1) Vedi la (4) del $\$ 3$.

(9) Vedi la (1) del $\$ 3$ con $j=2$. 
e, per le $(6)$ e $(7)$, osservando che $\zeta^{\prime}=\frac{d \zeta}{d \theta} \cdot \theta^{\prime}$,

e quindi la (j) diventa

$$
v^{2}=\left(\frac{d \zeta}{d \theta}\right)^{2} \cdot H^{2}+H^{2} \cdot \zeta^{2}
$$

$$
\left(\frac{d \zeta}{d \theta}\right)^{2}=-\zeta^{2}+2 b \zeta+a
$$

dove $b$ ed $a$ sono convenienti costanti.

La (8) si può serivere

$$
\left(\frac{d \zeta}{d \theta}\right)^{2}=-(\zeta-b)^{2}+c, \text { dove } \quad c=a+b^{2} .
$$

Da questa si ha, p. es.

dove

$$
d \theta=\frac{-d \xi}{\sqrt{1-\xi^{2}}}
$$

$$
\xi=(\zeta-b): \sqrt{c}
$$

Limitiamoci al caso in cui $c>0$ e quindi in cui $V \bar{c}$ è reale, ed integriamo. Abbiamo

con $\theta_{0}$ costante, e quindi

$$
\begin{gathered}
\theta-\theta_{0}=\arccos \xi, \\
\xi=\cos \left(\theta-\theta_{0}\right), \\
\zeta=b+\sqrt{c} \cdot \cos \left(\theta-\theta_{0}\right), \\
\rho=\frac{1}{\left[1+e \cos \left(\theta-\theta_{0}\right)\right] b}, \quad e=\frac{\sqrt{c}}{b} .
\end{gathered}
$$

Questa è l'equazione di. una conica della quale un fuoco è il polo del sistema di coordinate.

\section{$\S 5$. Un problema di moto in uno spazio sferico a 3 dimensioni.}

1. Consideriamo uno spazio euclideo a 4 dimensioni $S_{4}$ contenente l'origine $O$ dello spazio hilbertiano, ed indichiamo con

$$
\varphi_{1}, \varphi_{2}, \varphi_{3}, \varphi_{4}
$$

4 parametri normali e a 2 a 2 ortogonali appartenenti ad $S_{4}$. 
Consideriamo inoltre la varieta $V$ a 3 dimensioni che ha per determinante la punto-funzione

dove

$$
\begin{aligned}
& x_{1} \varphi_{1}+x_{2} \varphi_{2}+x_{3} \varphi_{3}+x_{4} \varphi_{4}, \\
& x_{1}=R \cdot(1-\cos u) \\
& x_{2}=R \cdot \operatorname{sen} u \cdot \cos v \\
& x_{3}=R \cdot \operatorname{sen} u \cdot \operatorname{sen} v \cdot \cos w \\
& x_{4}=R \cdot \operatorname{sen} u \cdot \operatorname{sen} v \cdot \operatorname{sen} w
\end{aligned}
$$

$R$, essendo una costante, ed $u, v, w$ essendo 3 coordinate curvilinee.

La $V$ è uno spazio sferico (a 3 dimensioni) contenuto in $S_{4}$, di raggio $R$ e di centro $R \varphi_{1}$.

2. Lo spazio lineare

$$
x_{1}=R \cdot h, \text { con }|h|<1,
$$

$(h$ costante)

taglia $V$ in una sfera, il cui raggio, è $R \cdot$ sen $\omega$, dove $\omega$ soddisfa alla relazione $1-\cos \omega=h$.

Questa sfera è il luogo dei punti di $V$ che hanno da $O$ una distanza geodetica $=R \omega$.

3. Imaginiamo che da $O$ si propaghi lungo alle geodetiche di $V$ un flusso che crei un campo $C$ di forze inversamente proporzionale alla superficie su cui si distribuisce e quindi proporzionale all'inverso del quadrato di $R \cdot \operatorname{sen} u$, e che le forze risultino tangenti a dette geodetiche e rivolte verso 0.

Poichè la posizione iniziale e la direzione della velocità iniziale del punto mobile individuano una sfera di $V$ per 0 , la quale è una vera varietá geodetica di $V$, ed è tale che tutte le forze del campo $C$ applicate ai suoi punti giacciono in essa, si vede che il problema del moto può essere studiato su questa sfera, whe possiamo imaginare coincidere colla $v=0$.

4. Abbiamo dunque una sfera $V^{\prime}$ di cui

è una determinante, dove

$$
\begin{aligned}
& x_{1} \varphi_{1}+x_{2} \varphi_{2}+x_{3} \varphi_{3} \\
& x_{1}=R \cdot(1-\cos u) \\
& x_{2}=R \cdot \operatorname{sen} u \cdot \cos v \\
& x_{3}=R \cdot \operatorname{sen} u \cdot \operatorname{sen} v,
\end{aligned}
$$

ed in essa un campo di forze di componenti

$$
F^{1}=\frac{-k^{2}}{R^{2} \operatorname{sen}^{2} u}, \quad F^{2}=0 .
$$


Le forze del campo ammettono il potenziale

$$
U=\frac{k^{2} \cot u}{R^{2}}
$$

Il quadrato dell' elemento lineare è dato da

$$
d s^{2}=R^{2}\left(d u^{2}+\operatorname{sen}^{2} u d v^{2}\right),
$$

e, nell' ipotesi $m_{i}=m_{a}$, l' equazione delle forze vive diventa:

$$
u^{\prime 2}+\operatorname{sen}^{2} u \cdot v^{\prime z}=2 \lambda^{2} \cot u+K
$$

dove $\lambda=k: R^{2}$ e $K$ è una couveniente costante.

La

diventa

$$
A^{2}=F^{2}
$$

da cui

$$
v^{\prime \prime}+2 \cot u \cdot u^{\prime} \cdot v^{\prime}=0
$$

$$
\log v^{\prime}+2 \log \operatorname{sen} u=\log H,
$$

dove $H$ è una costante, ed infine

$$
v^{\prime} \operatorname{sen}^{2} u=H .
$$

Sostituendo in (1) la $v^{\prime}$ che si ricava da (3), si ha

$$
u^{\prime 2}=K+2 \lambda^{2} \cot u-\frac{H^{2}}{\operatorname{sen}^{2} u},
$$

ed infine osservando che

$$
\frac{d u}{d v}=\frac{u^{\prime}}{v^{\prime}}=\frac{\operatorname{sen}^{2} u}{H} u^{\prime}
$$

si vede che, moltiplicando la (4) per $\frac{\operatorname{sen}^{4} u}{H^{2}}$, essa diventa

e, posto

$$
\left(\frac{d u}{d v}\right)^{2}=\frac{\left(K+2 \lambda^{2} \cot u-\frac{H^{2}}{\operatorname{sen}^{2} u}\right) \operatorname{sen}^{4} u}{H^{2}}
$$

$$
\zeta=\cot u, \quad \text { da } \text { cui } \quad d \zeta=\frac{-d u}{\operatorname{sen}^{2} u}
$$

$$
\left(\frac{d \zeta}{d v}\right)^{2}=M+2 N^{2} \zeta-\frac{1}{\operatorname{sen}^{2} u}
$$

dove $M=K: H^{2}, N=\lambda: H$. 
Ma

$$
\frac{1}{\operatorname{sen}^{2} u}=1+\cot ^{2} u
$$

e la $(\tilde{\jmath})$ diventa

$$
\left(\frac{d \zeta}{d v}\right)^{2}=M_{1}+2 N^{2} \zeta-\zeta^{2}
$$

con $M_{1}=M-1$.

Separando le variabili, si ha p. es.

da cui

$$
d v=\frac{-d \zeta}{\sqrt{-(\zeta-N)^{2}+\left(M_{1}+N^{2}\right)}},
$$

ossia

$$
\begin{gathered}
v=\arccos \frac{\zeta-N}{\sqrt{M_{1}+N^{2}}}+v_{0} \\
\zeta=N+\cos \left(v-v_{0}\right) \cdot \sqrt{M_{1}+N^{2}}
\end{gathered}
$$

$$
\operatorname{tg} u=\frac{a}{1+e \cos \left(v-v_{0}\right)}
$$

dove $a$ ed $e$ sono delle costanti.

$\mathrm{Si}$ vede allora che la traiettoria è una linea chiusa.

5. Il risultato ottenuto ci induce a fare alcune considerazioni :

a) Se si imagina che lo spazio ordinario sia uno spazio sferico contenuto in un $S_{4}$ e che sopra di esso i corpi materiali si attraggano secondo la legge di NewTon, o in altri termini se si imagina che l'azione di un punto materiale $A$ sopra un'altro punto materiale $B$ si traduca in una forza attrattiva diretta tangenzialmente in $B$ alla geodetica che congiunge $A$ con $B$, (circolo massimo) ed inversamente proporzionale all'area della superficie (sfera) dei punti che hanno da $A$ uguale distanza geodetica $\left({ }^{1}\right)$, sullo spazio sferico il moto di un pianeta si comporterà come nelle ipotesi classiche e non si presenterà spostamento di perielio.

b) L'ipotesi fatta alla lettera $a$ ) significa che la forza attraente di cui vi si parla è inversamente proporzionale al quadrato della distanza rettilinea (cioè distanza nell' $S_{4}$ ) di $B$ da $A$.

c) Si sa che EINsTEIN ha dato una formula che spiega lo spostamento del perielio di Mercurio $\left({ }^{2}\right)$. In questa formula la forza attrattiva dei corpi è

(1) Forza calcolata al primo passaggio dell azione pel punto $B$.

(2) T. Levi-CivitA, Fondamenti di Meccanica Relativistica (ed. Zanichelli, 1928, p. 123). 
la somma di due, rispettivamente inversamente proporzionali al quadrato e al cubo della distanza del corpo attraente al corpo attratto. Le cose andrebbero in modo analogo se si pensasse esistere uno spazio fisico $E$ lineare e a 4 dimensioni e che lo spazio materiale $M$ (quello in cui la materia si deve muovere) sia uno spazio sferico a 3 dimensioni immerso in $F$, se inoltre si pensasse che da un corpo materiale escano due forze, la prima che si propaga soltanto in $M$ e che si diffonda su sfere concentriche, e che sarà naturale pensare inversamente proporzionale al quadrato della distanza, la seconda che si propaga in $F$ e che si diffónda su spazi sferici a 3 dimensioni concentrici, e che sarà naturale pensare inversamente proporzionale al cubo della distanza. Probabilmente le cose non saranno cosi, e nemmanco sarà possibile su questo indirizzo imbastire una teoria che soddisfi il nostro spirito. Tuttavia puó valer la pena che io abbia esposta questa considerazione. 